Reprod. Nutr. Dévelop., 1981, 21 (1), 47-57.

\title{
Effets de l'énucléation oculaire bilatérale sur l'ultrastructure de l'épiphyse chez la femelle du Lérot (Eliomys quercinus L.). Corrélations avec l'axe hypothalamo-hypophyso-ovarien
}

\author{
par Michelle ROUX, J. P. RICHOUX \\ avec la collaboration technique de Bernadette CUNIN
}

Laboratoire d'Hisfologie, Faculté de Médecine A, B.P. 184, 54500 Vandœuvre lès Nancy, France.

Summary. Effects of blinding by bilateral orbital enucleation on the ultrastructure of the pineal gland in the female garden dormouse (Eliomys quercinus L.). Correlations with the hypothalamo-pituitary-ovarion axis.

Dormice were blinded by bilateral enucleation in December before hibernation or in March at the end of hibernation and were then studied in July and October. All the animals stayed in permanent anoestrus even at long-term. The ultrastructure of the pinealocyte was very characteristic, presenting typical elements of both hibernal anoestrus and sexual activation. Two organelles were well developed in enucleated dormice : reticulo-endoplasmic vacuoles and liposomes whose development seemed to be associated with protein secretions and pineal serotonine level, respectively. The hypothalamo-hypophyseal characterististics of the enucleated animals were also surprising : the hypothalamic LHRH load was important and hypophyseal gonadotropic cell development normal although the ovaries were hypoactive. The dormouse has a seasonal sexual cycle which cannot be realized without light. Pineal ultrastructural findings correlated with the activity of the hypothalamo-pituitary-ovarian axis lead us to think that this endocrine gland plays a role in the action of light on sexual activity.

\section{Introduction.}

L'éfude des relations d'activité entre l'épiphyse ef la fonction reproductrice chez les Mammifères a donné lieu à de nombreux travaux (revues de Kappers, 1969, 1976 ; Reiter, 1978). Cependant, la nature des principes actifs épiphysaires, leur localisation ultrastructurale, leur mode d'action ne sont pas clairement définis. Par des études en microscopie électronique nous avons essayé de mettre en évidence des caractéristiques morphologiques de l'état fonctionnel du pinéalocyte au cours du cycle sexuel saisonnier chez la femelle d'un hibernant, le Lérot. Nous avons ainsi pu montrer que, pendant la période d'activité génitale comme pendant la phase de repos hivernal, il existait une relation entre l'aspect ultrastructural du pinéalocyte, le mode d'éclairement ef l'état fonctionnel de l'appareil génital (Roux et Richoux, 1975 ; Richoux et al., 1975 ; Roux et al., 1977, 1979). Des organites pinéalocytaires caractéristiques d'un état génital donné apparaissent en général avant ce dernier ; cette observation apporte 
un argument en faveur de l'hypothèse de Reiter (1974) selon laquelle la durée d'illumination déterminerait directement l'état fonctionnel de la pinéale et secondairement celui des gonades. Pour compléter nos résultats, nous avons étudié l'effet de l'énucléation bilałérale réalisée en décembre avant la période hivernale et à la mi-mars dès la sortie de l'hibernation, chez des animaux sacrifiés respectivement en juillet et en octobre.

\section{Matériel et méthodes.}

Une vingtaine d'animaux ont été étudiés et classés en 5 lots :

1. - Animaux témoins, vivant au laboratoire à $22^{\circ} \mathrm{C}$ avec une photopériode $L-D$ : 10-14 ( $\mathrm{L}: 8 \mathrm{~h}-18 \mathrm{~h})$ de décembre à fin juin-début juillet.

2. - Animaux énucléés bilatéralement en décembre, vivant au laboratoire à $22^{\circ} \mathrm{C}$ avec une photopériode L-D : 10-14 ( $L: 8 \mathrm{~h}-18 \mathrm{~h}$ ), sacrifiés en juillet.

3. - Animaux énucléés bilatéralement en décembre, vivant au laboratoire à $22^{\circ} \mathrm{C}$, maintenus en illumination continue, sacrifiés en juillet.

4. - Animaux témoins, hibernant au laboratoire dans des conditions proches de la vie naturelle : maintien en chambre froide à $6^{\circ} \mathrm{C}$ et à l'obscurité du 15 décembre au 15 mars, ensuite à $22^{\circ} \mathrm{C}$ sous une photopériode $L-D: 10-14(L: 8 \mathrm{~h}-18 \mathrm{~h}$ ), de mars à octobre.

5. - Animaux hibernant au laboratoire de décembre à mars, ces animaux énucléés bilatéralement le jour même de leur sortie d'hibernation expérimentale ont été maintenus ensuite à $22^{\circ} \mathrm{C}$ sous un régime lumineux $L-D: 10-14(\mathrm{~L}: 8 \mathrm{~h}-18 \mathrm{~h})$ et sacrifiés en octobre.

Les animaux ont été décapités sans anesthésie ; l'épiphyse prélevée rapidement a été fixée par immersion dans du glutaraldéhyde-formol (glutaraldéhyde 1,8 p. 100, paraformaldéhyde $0,7 \mathrm{p}$. 100 tampon phosphate $0,1 \mathrm{M}$, $\mathrm{pH} \mathrm{7,4,} \mathrm{to} \mathrm{:} 4{ }^{\circ} \mathrm{C}$ ) pendant $1 \mathrm{~h}$, post-fixée au tétroxyde d'osmium pendant $1 \mathrm{~h}\left(\mathrm{O}_{\mathrm{S}} \mathrm{O}_{4} 1 \mathrm{p} .100\right.$, tampon phosphate $0,1 \mathrm{M}, \mathrm{pH} 7,4, t^{\circ}: 4^{\circ} \mathrm{C}$ ), puis déshydratée dans les alcools, l'oxyde de propylène ef incluse dans l'araldite.

Des coupes ultrafines recueillies sur grilles ont été contrastées par l'acétate d'uranyle, le citrate de plomb ef observées au microscope électronique Siemens 101.

\section{PLANCHE I}

FIG. 1. - Pinéalocyfes de Lérof témoin, en activité génitale cyclique (fin juin-début juillet) : mitochonidries à crêtes de petite taille, appareil de Golgi et ergastoplasme bien développés ; nombre élevé de ribosomes groupés en polysomes, absence de liposomes $(\times 27000)$.

FIG. 2. - Pinéalocyte de Lérof énucléé en décembre et sacrifié en juillef : appareil de Golgi et ergastoplasme bien développés, nombreux ribosomes groupés en polysomes, vacuoles $(\times 36000)$.

Abréviations : $\mathrm{m}$ : mifochondrie ; $\mathrm{g}$ : appareil de Golgi ; e : ergastoplasme ; $r$ : ribosome ; $v$ : vacuole ; li : liposome ; ly : lysosome.

Grossissement : la longueur de la barre représente $1 \mu \mathrm{m}$ à l'échelle de la figure. 


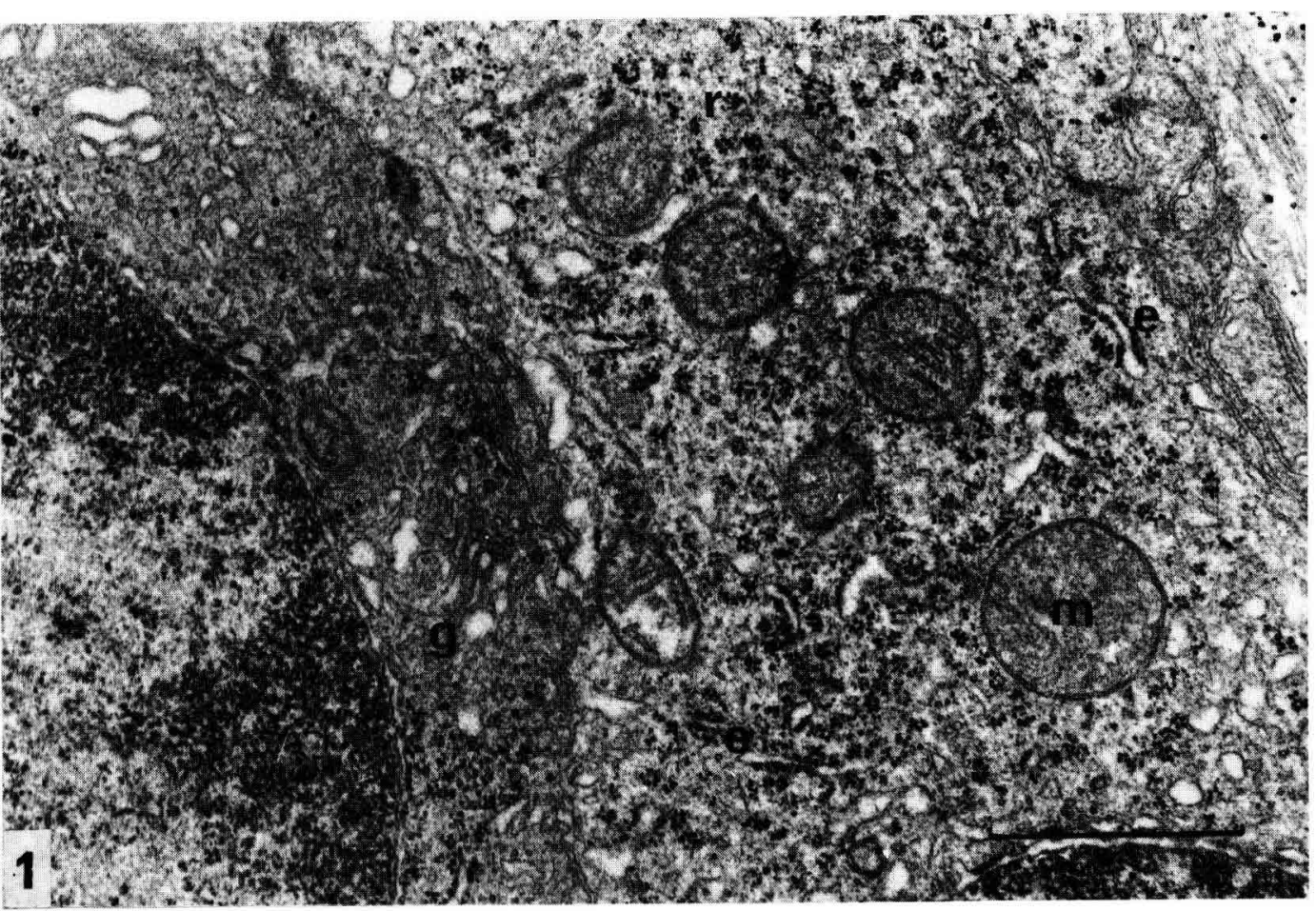

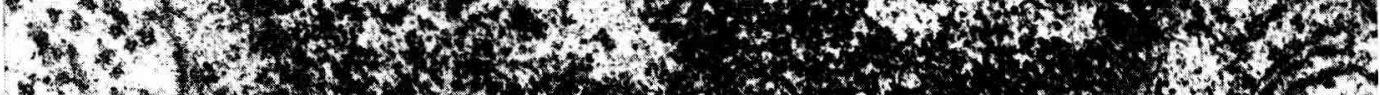

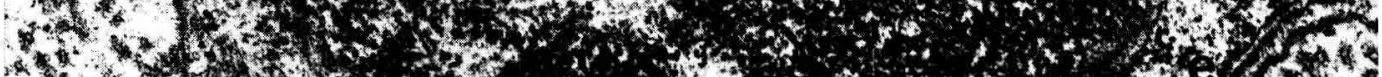

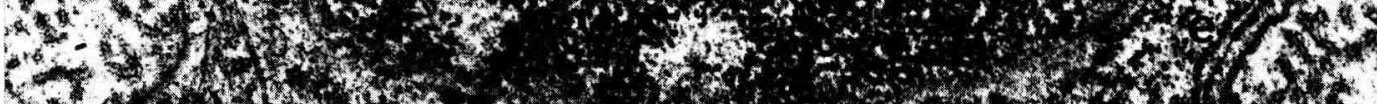

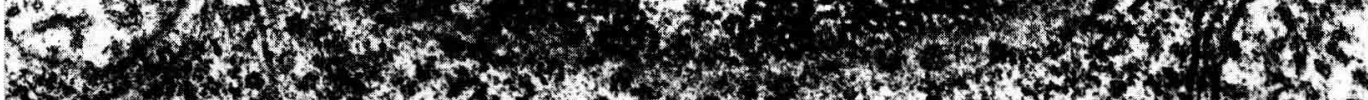

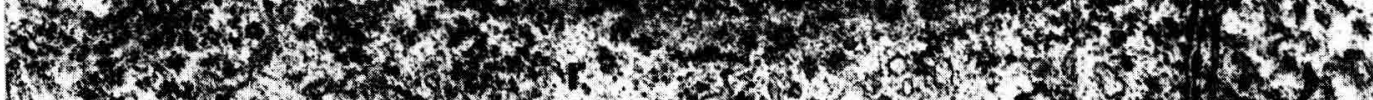
H.

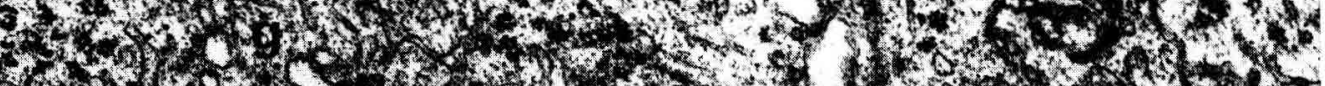

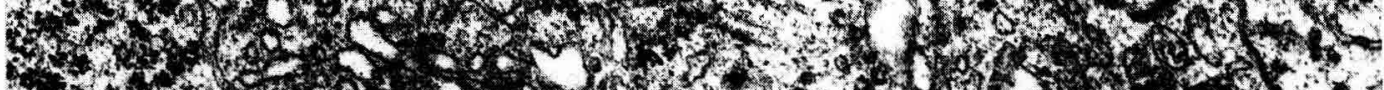

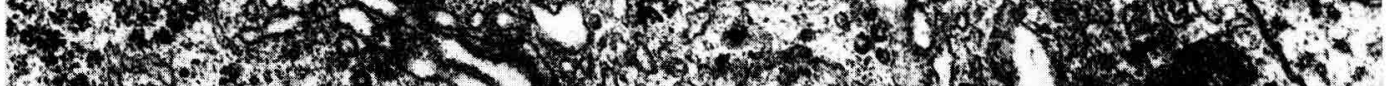
(2)

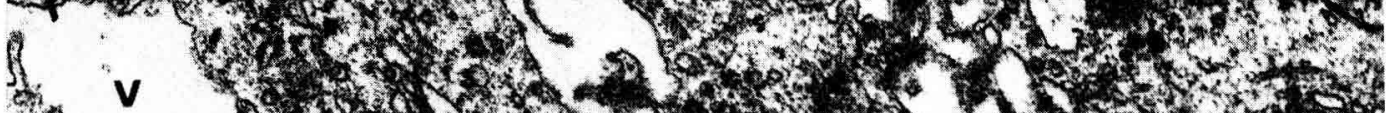

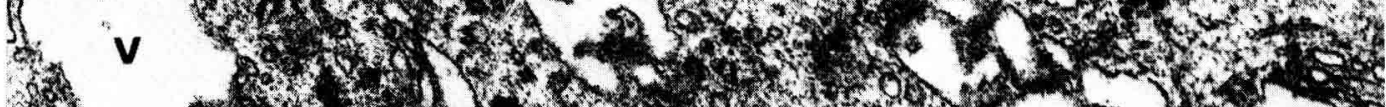
6.

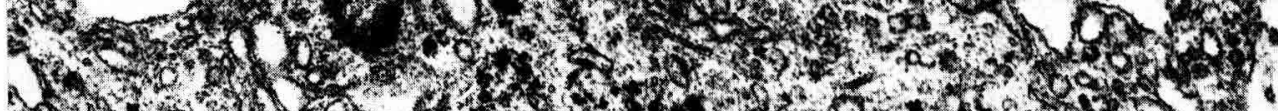

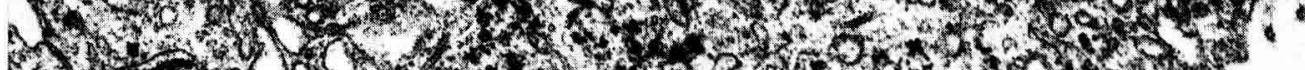

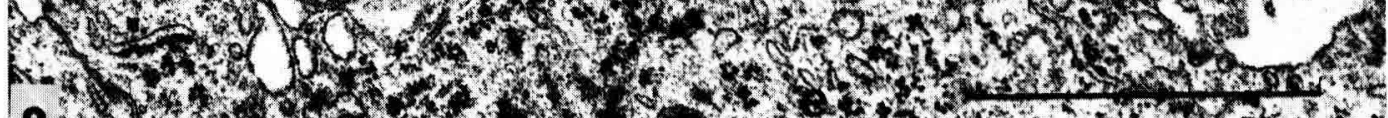

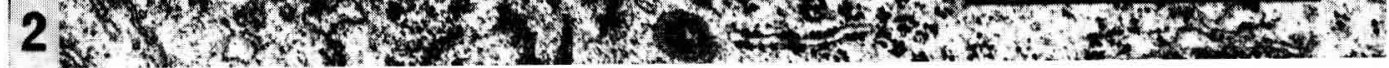




\section{Résulfats.}

A. - En juillet, l'épiphyse des animaux témoins (loł 1) a la structure typique, déjà décrite précédemment, de celle d'animaux sexuellement actifs (Roux et al., 1977). Le pinéalocyte présente un cytoplasme dense ; l'appareil de Golgi et l'ergastoplasme sont bien développés. Le nombre de ribosomes libres isolés ou groupés en polysomes est élevé. Les mitochondries à crêtes parallèles sont de petite taille $(0,5 \mu \mathrm{m})$. On rencontre fréquemment des grains de sécrétion à cœur dense à proximité de l'appareil de Golgi ef des rubans circonscrits par des vésicules (RCV) dans le périkaryon ou près de la membrane cellulaire ; les liposomes sont peu nombreux ou absents, les lysosomes très rares (fig. 1).

Tous ces animaux sont en activité génitale cyclique.

Le pinéalocyte des Lérołs énucléés en décembre et observés en juillet (lot 2 et lot 3 ) présente une ultrastructure comparable quel que soit le mode d'éclairement des animaux. Il a, à la fois, les caractéristiques des animaux témoins : appareil de Golgi et ergastoplasme bien développés, mitochondries à crêtes de petite faille, diminution du nombre des liposomes (fig. 2), et celles des animaux en anœstrus hivernal : existence de nombreuses vacuoles, de taille variable, délimitées par une membrane lisse (fig. 3) ; malgré la diminution du nombre de liposomes, on voit des accumulations de ces organites associés étroitement à d'importantes formations lysosomiques très osmiophiles (fig. 4). Les grains de sécrétion à cour dense ef les RCV sont rares. Ces animaux sont demeurés en ancestrus.

B. - Les animaux témoins d'octobre (lot 4) ont une structure pinéalocytaire très différente de celle du mois de juillet. L'appareil de Golgi et l'ergastoplasme sont toujours bien développés ; les mitochondries à crêtes parallèles ont une taille moyenne; il y a apparition de vacuoles (fig. 5), de liposomes (fig. 6) et de formations lysosomiques. Les grains de sécrétion et les RCV sont très peu nombreux. Le pinéalocyte a un aspect de transition entre celui de la période d'activité génitale et celui de la phase hivernale. Après une période d'activité génitale normale (mi-avril, mi-juillet), ces animaux sont de nouveau en anœstrus.

Chez les animaux énucléés à la sortie de l'hibernation et sacrifiés en octobre (lot 5), l'aspect ultrastructural du pinéalocyte est semblable à celui des Lérots énucléés, observés en juillet et très peu différent de celui des témoins de cette époque ; soulignons la fréquence des formations vacuolaires (fig. 7) et l'association de liposomes et de lysosomes (fig. 8). Ces animaux sont demeurés en anœstrus.

\section{PLANCHE II}

FIG. 3. - Pinéalocyte de Lérot énucléé en décembre et sacrifié en juillet : nombreuses vacuoles de nature réticulo-endoplasmique $(\times 27500)$.

FIG. 4. - Pinéalocyte de Lérot énucléé en décembre et sacrifié en juillet : accumulation de liposomes associés à des formations lysosomiales $(\times 25300)$. 


\section{Discussion.}

Dans un travail précédent (Roux et al., 1977), nous avions éfudié l'ultrastructure de l'épiphyse chez des animaux placés dans diverses conditions d'éclairement. Chez les Lérots maintenus à l'obscurité permanente de décembre à fin mai-début juin, le pinéalocyte présentait les caractéristiques essentielles de celui de l'animal hibernant ; nous avions déjà noté le développement particulier des vacuoles intra-cellulaires que nous retrouvons chez les animaux énucléés. Le conditionnement en chambre obscure induit l'ancestrus permanent. L'énucléation bilatérale a les mêmes effets à court ou à long terme ; l'illumination continue ne modifie pas l'effet de l'énucléation.

L'ultrastructure du pinéalocyte est plus complexe dans notre nouvelle expérimentation : elle est véritablement « hybride » entre l'aspect typique rencontré, d'une part, chez les animaux en période d'activité génitale par le développement de l'appareil de Golgi, de l'ergastoplasme, la présence de mitochondries à crêtes de petite taille et, d'autre part, chez les animaux en ancstrus hivernal par la richesse en vacuoles, l'accumulation de liposomes, bien que réduits en nombre, et en général associés à des lysosomes.

Or les caractéristiques hypothalamo-hypophysaires de l'état d'ancstrus induit par l'énucléation sont également bien surprenantes : chez les animaux énucléés sacrifiés en juillet ou en octobre, les observations immunocytologiques ef les dosages radioimmunologiques montrent une mise en charge importante de LHRH hypothalamique ; les cellules gonadotropes antéhypophysaires sont bien différenciées et ont un développement normal. L'ovaire demeurant inactif, il est probable que l'excrétion du LHRH dans le système porte soit perturbée et la sécrétion des hormones gonadotropes très faible (Richoux, en préparation).

Notons que les animaux témoins d'octobre dont l'éclairement est réduit progressivement depuis juillet, et qui sont à nouveau en anœstrus, ont également une charge en LHRH hypothalamique très augmentée par rapport à celle des témoins de juillet ef des cellules gonadotropes encore bien développées (Richoux, en préparation). Une dissociation similaire entre l'état d'activité de l'axe hypothalamo-hypophysaire (forte charge en LHRH hypothalamique, taux élevé de la LH pituitaire) ef de celui des gonades (repos génital) a été observée chez le Rat énucléé et anosmique par Johnson et Reiter (1978) et chez la femelle du Hamster privée de lumière par Reiter (1978).

\section{PLANCHE III}

FIG. 5. - Pinéalocyte de Lérot témoin, sacrifié en octobre : présence de nombreuses vacuoles de nałure réticulo-endoplasmique $(\times 20000)$.

FIG. 6. - Pinéalocyie de Lérof témoin, sacrifié en octobre : apparition de liposomes ( $\times 22700$ ).

FIG. 7. - Pinéolocyfe de Lérot énucléé en mors ef sacrifié en octobre : appareil de Golgi et ergastoplasme bien développés; dilation réticulo-endoplasmique ( $\times 27400)$.

FIG. 8. - Pinéalocyte de Lérot énucléé en mars et sacrifié en octobre : présence de liposomes et lysosomes $(\times 30000)$. 
La pinéalectomie annulant les effets de la privation de lumière et de l'anosmie sur les gonades, ces auteurs envisagent une action inhibitrice de l'épiphyse sur la sécrétion de $\mathrm{LHRH}$ et de $\mathrm{LH}$; son intervention directe au niveau même de l'ovaire est avancée par Reiter (1978).

Si l'absence de lumière agit par l'intermédiaire de l'épiphyse pour empêcher la réalisation des potentialités gonadotropes du complexe hypothalamo-antéhypophysaire, l'ultrastructure si particulière du pinéalocyłe des animaux énucléés pourrait fournir des arguments aidant à élucider les processus fonctionnels de cette glande endocrine. Il est actuellement admis que le pinéalocyte des Mammifères élabore des indolamines et des composés peptidiques. La sérotonine et ses dérivés méthoxyindoliques (mélatonine, 5-méthoxytryptophol...) sont synthétisés selon un mécanisme enzymatique ; les composés peptidiques, non encore identifiés, sont élaborés selon un procédé ribosomal classique. La localisation ultrastructurale de ces deux types de substance n'est cependant pas nettement définie, leurs relations fonctionnelles dans le pinéalocyte restent hypothétiques (revue de Collin, 1979).

L'organite dont le développement est le plus remarquable chez les animaux en ancestrus est le liposome ; nous avions déjà souligné ce fait chez le Lérot hibernant (Richoux et al., 1975) et le Lérot privé de lumière (Roux et al., 1977). Rappelons que les liposomes sont fortement réduits en nombre et en taille chez l'animal en activité génitale et réapparaissent en automne quand la régression ovarienne a lieu. Le pinéalocyte des animaux énucléés n'est pas aussi riche en lipides que celui des hibernants ; par ailleurs, les formations lysosomiales particulièrement osmiophiles, associées aux liposomes ef ressemblant aux granules de lipofuchsine, sont un signe évident de leur dégradation chez ces animaux. Ces images traduisent-elles le blocage d'un processus sécrétoire à effet antigonadotrope devenu superflu chez ces animaux en anœstrus permanent?

Si les liposomes ont été décrits dans l'épiphyse de la plupart des Mammifères étudiés (Prop, 1965 ; Pevet, 1979), on ne connaît pas actuellement la nature précise de leur contenu. Chez le Lérot hibernant, les liposomes nombreux et de grande taille sont fréquemment associés à des mitochondries de type tubulaire (Roux et al., 1977) ; celles-ci sont en général impliquées dans l'élaboration de stéroïdes; cependant, Sheridan ef Reiter (1968) ont montré, chez le Hamster, que la pinéale ne renfermait pas de stéroïdes. Prop (1965) signale avoir mis en évidence, dans les liposomes de l'épiphyse du Rat, des triglycérides et des phospholipides. Nous avons constaté, pendant la période hivernale, que le développement des liposomes était parallèle à la richesse de l'épiphyse en indolamines (Cordonnier ef Roux, 1978). D'autres auteurs ont observé cette association (Quay, 1961 ; Perrelet et al., 1968). Perrelet et al. suggèrent même la présence d'indolamines dans les liposomes. Arstila et al. (1971), après incorporation de précurseurs tritiés de la sérotonine dans une culture de tissu épiphysaire de Rat, signalent une localisation de radioactivité au niveau des liposomes des pinéalocytes. Il n'est pas exclu que ces organites puissent servir de « magasin » à la sérotonine ou à d'autres indolamines. Si la relation exacte entre liposomes et indolamines épiphysaires n'est pas clarifiée, nous avons cependant constaté que le développement de ces deux éléments était associé à un état d'hypoactivité génitale.

Nos observations, ainsi que celles d'autres auteurs sur les variations de l'importance des liposomes en fonction des conditions d'éclairement (Quay, 1961), des phases 
du cycle cestrien (Zweens, 1963), du taux d'hormone gonadotrope circulante (Zweens, 1965 ; Karasek et Marek, 1978) nous font penser, avec Pevet (1979), que ces organites sont impliqués dans les processus sécrétoires de la pinéale. Dès 1965, Prop attribue aux lipides un rôle dans le métabolisme général de l'épiphyse, peut-être en tant que source d'énergie pour les différents processus de synthèse.

Un deuxième organite particulièrement développé chez les animaux énucléés est représenté par les vacuoles qui ont la structure de dilatations réticulo-endoplasmiques. Nous avions également signalé ces éléments chez le Lérot hibernant et le Lérot maintenu à l'obscurifé (Roux et al., 1977). Rappelons que tous ces animaux sont en état d'anœstrus. Des vacuoles intracellulaires semblables ont été observées dans l'épiphyse de divers Mammifères (revue de Pevet, 1979). Pevet et Smith (1975) trouvent ces formations en grand nombre chez la Taupe en procstrus. Ces vacuoles dont l'extension peut être considérable font penser aux vacuoles de castration des cellules gonadotropes antéhypophysaires et pourraient comme ces dernières, ainsi que le suggère Pevet, accumuler des produits actifs. Une relation fonctionnelle entre ces organites et l'activité hypothalamo-hypophyso-génitale paraît vraisemblable; cependant, ni la nature, ni le rôle du produit accumulé dans leur cavité ne sont connus. D'après les données sur les processus cellulaires de la synthèse protéique (Palade, 1975), on peut envisager l'accumulation dans ces vacuoles de substances polypeptidiques directement ou indirectement reliées au métabolisme d'un principe actif de la pinéale.

Les grains à cœur dense situés à proximité de l'appareil de Golgi sont considérés par la plupart des auteurs comme des grains de sécrétion (revues de Collin, 1979, de Pevet, 1979). Collin (1979), Juillard et Collin (1980) ont montré chez deux Sauropsidés et un Mammifère que ces organites étaient le lieu de stockage à la fois de matériel protéique et de sérotonine. D'importantes variations de leur nombre ont été observées dans diverses conditions expérimentales relatives à la photopériode (Pevet, 1979). Chez le Lérot, le nombre de grains de sécrétion augmente pendant la période d'activiłé génitale et chez les animaux qui manifestent une activation ovarienne en hiver après illumination continue (Roux et al., 1977 ; Cordonnier et Roux, 1978). Ces organites sont rares chez les Lérots en état d'ancestrus : animaux hibernants, privés de lumière ou énucléés. Par ailleurs, chez celte espèce, le nombre de grains de sécrétion n'est pas proportionnel à la richesse de la pinéale en sérotonine (Cordonnier et Roux, 1978). Le rôle de ces organites reste inconnu ; une action antigonadotrope de leur contenu a souvent été avancée (Pevet, 1979) ; il faudrait admettre une excrétion rapide de ces grains chez les Lérots en anœstrus pour interpréter nos observations dans ce sens.

Les rubans circonscrits par des vésicules (RCV), bien développés en nombre chez le Lérot pendant la période d'activité génitale printanière quand la luminosité naturelle s'intensifie et chez les animaux illuminés en période hivernale (Roux ef al., 1977 ; Cordonnier et Roux, 1978), sont très rares chez les animaux en anœstrus induit par énucléation ou chez les hibernants. Des variations de leur nombre et de leur taille onf été observées en fonction des conditions d'éclairement, de l'importance de l'innervation catécholaminergique, de l'étał génital, d'agressions diverses chez de nombreuses espèces (Pevet, 1979). Si une signification fonctionnelle de ces organites est vraisem- 
blable, leur participation directe aux processus sécrétoires du pinéalocyte paraît exclue ; un rôle de transmission entre pinéalocytes est le plus souvent avancé (Pevet, 1979).

\section{Conclusion.}

Des Lérots énucléés bilatéralement, soit en décembre avant la période hivernale, ou à la mi-mars dès la sortie de l'hibernation, restent en anœstrus permanent, même à long terme.

Si ces animaux ont un complexe hypothalamo-hypophysaire à compétences gonadotropes évidentes, celles-ci ne peuvent pas se réaliser sans lumière ; en effel chez quelques Lérots énucléés 15 à 20 jours après le réveil printanier, nous avons constaté que cette courte période de lumière après le repos hivernal suffisait pour permettre la réalisation de la programmation de l'activité génitale. Cette espèce ne présente donc pas de réveil génital printanier spontané, indépendant des conditions d'éclairement préalables, ainsi que le montre Reiter (1978) chez le Hamster.

L'ultrastructure de l'épiphyse des Lérots énucléés dont le pinéalocyte a un aspect très particulier, «hybride » entre celui de l'animal en activité génitale et celui de l'hibernant en anœstrus, apporte de nouveaux arguments en faveur de l'intervention de la glande pinéale dans l'action de la lumière sur l'activité génitale. Deux organites ont particulièrement frappé notre attention: les liposomes dont le développement paraît être associé à la richesse de la pinéale en sérotonine et les vacuoles de nature réticulo-endoplasmique qui pourraient stocker des protéines.

II est probable que l'épiphyse synthétise plusieurs substances actives pro- et/ou antigonadotropes; chez le Lérot les organites pinéalocytaires, caractéristiques de l'état d'activité génitale ou de l'anœstrus, semblent bien être impliqués dans des processus sécrétoires différents.

Reçu en avril 1980

Accepté en septembre 1980.

Remerciements. - Ce travail a été réalisé avec l'aide de la DGRST (Contrat no 77.7.0662 : Biologie de la Reproduction et du Développement).

Les observations ultrastructurales ont été faites au laboratoire de Microscopie électronique Faculté de Médecine de Nancy, grâce à l'obligeance de Monsieur le Professeur G. Grignon.

\section{Références}

ARSTILA A. U., KALIMO H. O., HYYPPÄ M., 1971. Secretory organelles of the rat pineal gland : electron microscopic and histochemical studies in vivo and in vitro, 147-164. In WOLSTENHOLME G. E. W., KNIGHT J., The pineal gland (A Ciba Foundation Symposium), Churchill Livingstone, Edinburgh-London.

COLLIN J. P., 1979. Recent advances in pineal cytochemistry. Evidence of the production of indoleamines and proteinaceous substances by rudimentary photoreceptor cells and pinealocytes of amniota, 271-296. In KAPPERS J. ARIËNS, PEVET P., The pineal gland in vertebrates including man. Progr. Brain Res., vol. 52, Elsevier, Amsterdam. 
CORDONNIER J. L., ROUX M., 1978. Ełude en histofluorescence des monoamines épiphysaires chez le Lérot (Eliomys quercinus $L$.) dans diverses conditions expérimentales au cours de la période hivernale. Ann. End., 39, 403-410.

JOHNSON L. Y., REITER R. J., 1978. The pineal gland and its effects on mammalian reproduction. Prog. reprod. Biol., 4, 116-156.

JUILLARD M. T., COLLIN J. P., 1980. Les pinéalocytes (cellules récepto-sécrétrices) de la glande pinéale de Mammifères : éfude chez la Souris. Biol. cell., 38, $2 a$.

KAPPERS J. ARIËNS, 1969. The mammalian pineal organ. J. neuro-visc. Rel., suppl. 9, 140-184.

KAPPERS J. ARIËNS, 1976. The mammalian pineal gland, a survey. Acta neurochir., 34, 109-149.

KARASEK M., MAREK K., 1978. Influence of gonadotropic hormones on the ultrastructure of rat pinealocytes. Cell Tiss. Res., 188, 133-141.

PALADE G., 1975. Intracellular aspects of the process of protein synthesis. Science, 189, 347-358.

PERRELET A., ORCI M., ROUILLER Ch., 1968. Clarification of the osmiophilic granules of the rat pinealocytes by P-chlorophenylalanin. Experientia, 26, 1047-1049.

PEVET P., 1979. Secretory processes in the mammalian pinealocyte under natural and experimental conditions, 149-194. In KAPPERS J. ARIËNS, PEVET P. The pineal gland in vertebrates including man. Progr. Brain Res., vol. 52, Elsevier, Amsterdam.

PEVET P., SMITH A. R., 1975. The pineal gland of the mole (Talpa europaea L.). Il. Ultrastructural variations observed in the pinealocytes during different parts of the sexual cycle. J. neural Transm., 36, 227-248.

PROP N., 1965. Lipids in the pineal body of the rat, 454-464. In KAPPERS J. ARIËNS, SCHADÉ J. P., Structure and function of the epiphysis cerebri. Progr. Brain Res., vol. 10, Elsevier, Amsterdam.

QUAY W., 1961. Reduction of mammalian pineal weight and lipids during continuous light. Gen. comp. Endocr., 1, 211-217.

REITER R. J., 1974. Pineal-anterior pituitary gland relationships. M. T. P. Int. Rev. Sci., Endocr. Physiol., 5, 277-305.

REITER R. J., 1978. Interaction of photoperiod, pineal and seasonal reproduction as exemplified by findings in the hamster. Prog. reprod. Biol., 4, 169-190.

RICHOUX J. P., ROUX M., LEGAIT H., 1975. Etude de l'appareil génital femelle et de l'épiphyse du Lérot dans diverses conditions au cours de la période hivernale. J. Physiol., 70, 3, 21 B.

ROUX M., RICHOUX J.P., 1975. Etude ultrastructurale de l'épiphyse du Lérot (Eliomys quercinus L.) vivant dans des conditions normales et expérimentales. J. Microsc. Biol. cell., 22, 33-34a.

ROUX M., RICHOUX J. P., CORDONNIER J. L., 1977. Influence de la photopériode sur l'ultrastrucfure de l'épiphyse avant et pendant la phase génitale saisonnière chez le Lérot (Eliomys quercinus L.). J. neural Transm., 41, 209-223.

ROUX M., CORDONNIER J. L., RICHOUX J. P., 1979. Ultrastructural and monoamine histofluorescence study of pineal gland in the female garden dormouse (Eliomys quercinus $L$.) under normal and experimental conditions during the period of hibernation. Correlations with the hypothalamo-pituitary-ovarian axis, 201-206. In KAPPERS J. ARIËNS, PEVET P., The pineal gland in vertebrates including man. Progr. Brain Res. vol. 52, Elsevier, Amsterdam.

SHERIDAN M. N., REITER R. J., 1968. The fine structure of the hamster pineal gland. Amer. J. Anat., 122, 357-376.

ZWEENS J., 1963. Influence of the oestrus cycle and ovariectomy on the phospholipid content of the pineal gland in the rat. Nature (cond.), 197, 1114-1115.

ZWEENS J., 1965. Alterations of the pineal lipid content in the rat under hormonal influences, 540551. In KAPPERS J. ARIENS, SCHADE J. P., Structure and function of the epiphysis cerebri. Progr. Brain Res. vol. 10, Elsevier, Amsterdam. 